\title{
Efficacy and cost of video-assisted thoracoscopic partial pleurectomy versus talc pleurodesis in patients with malignant pleural mesothelioma (MesoVATS): an open-label, randomised, controlled trial
}

Robert C Rintoul, Andrew J Ritchie, John G Edwards, David A Waller, Aman S Coonar, Maxine Bennett, Eleonora Lovato, Victoria Hughes, Julia A Fox-Rushby, Linda D Sharples, on behalf of the MesoVATS Collaborators*

\section{Summary}

Background Malignant pleural mesothelioma incidence continues to rise, with few available evidence-based therapeutic options. Results of previous non-randomised studies suggested that video-assisted thoracoscopic partial pleurectomy (VAT-PP) might improve symptom control and survival. We aimed to compare efficacy in terms of overall survival, and cost, of VAT-PP and talc pleurodesis in patients with malignant pleural mesothelioma.

Methods We undertook an open-label, parallel-group, randomised, controlled trial in patients aged older than 18 years with any subtype of confirmed or suspected mesothelioma with pleural effusion, recruited from 12 hospitals in the UK. Eligible patients were ro nly assigned (1:1) to either VAT-PP or talc pleurodesis by computer-generated random numbers, stratified by E ean Organisation for Research and Treatment of Cancer risk category (high vs low). The primary outcome was overall survival at 1 year, analysed by intention to treat (all patients randomly assigned to a treatment group with a final diagnosis of mesothelioma). This trial is registered with ClinicalTrials.gov, number NCT00821860.

Findings Between Oct 24, 2003, and Jan 24, 2012, we randomly assigned 196 patients, of whom 175 (88 assigned t pleurodesis, 87 assigned to VAT-PP) had confirmed mesothelioma. Overall survival at 1 year was $52 \%$ (95\% CI 41-62) in the VAT-PP group and 57\% (46-66) in the talc pleurodesis group (hazard ratio 1.04 [95\% CI 0 -76-1.42]; p=0 81). Surgical complications were significantly more common after VAT-PP than after talc pleurodesis, occurring in $24(31 \%)$ of 78 patients who completed VAT-PP versus ten (14\%) of 73 patients who completed talc pleurodesis ( $\mathrm{p}=0.019)$, as were respiratory complications (19 [24\%] vs 11 [15\%]; $\mathrm{p}=0 \cdot 22$ ) and air-leak beyond 10 days (five [6\%] vs one $[1 \%] ; p=0 \cdot 21$ ), although not significantly so. Median hospital stay was longer at 7 days (IQR 5-11) in patients who received VAT-PP compared with 3 days $(2-5)$ for those who received talc pleurodesis $(p<0 \cdot 0001)$.

Interpretation VAT-PP is not recommended to improve overall survival in patients with pleural effusion due to malignant pleural mesothelioma, and talc pleurodesis might be preferable considering the fewer complications and shorter hospital stay associated with this treatment.

Funding BUPA Foundation.

\section{Introduction}

The incidence of malignant pleural mesothelioma continues to rise; ${ }^{1-3}$ however, few evidence-based therapeutic options are available. Cisplatin plus pemetrexed chemotherapy has been shown to confer a slight survival advantage. ${ }^{4,5}$ The role of surgical resection is uncertain, but several operative approaches have been described. ${ }^{6}$ Extrapleural pneumonectomy (EPP) has been assessed in several studies-most recently in the MARS trial ${ }^{7}$ as part of trimodality therapy. Lung-sparing approaches, including (extended) pleurectomy with decortication $^{8-10}$ and video-assisted thoracoscopic partial pleurectomy (VAT-PP), have also been assessed, ${ }^{11,12}$ and might be particularly appropriate for patients with more advanced disease or comorbidities.

VAT-PP involves thoracoscopic debulking of the parietal pleura and visceral pleurectomy with decortication to release trapped lung. ${ }^{6}$ Results of non-
${ }^{*}$ Members listed at end of report Department of Thoracic Oncology, Papworth Hospital Cambridge, UK (RC Rintoul FRCP, $V$ Hughes PhD Prof LD Sharples PhD); Essex Cardiothoracic Centre, Basildon, UK (A J Ritchie FRCS); Department of Cardiothoracic Surgery, Northern General Hospital, Sheffield, UK (J G Edwards FRCS [CTh]); Department of Thoracic Surgery, Glenfield Hospital, Leicester, UK

(D A Waller FRCS [CTh]); Department of Thoracic Surgery, Papworth Hospital, Cambridge, UK (A S Coonar FRCS [CTh]) MRC Biostatistics Unit, Cambridge, UK (M Bennett MSC, Prof L D Sharples); Health Economics Research Group, Brunel University, Uxbridge, UK (E Lovato MSc,

ProfJ A Fox-Rushby PhD); and Clinical Trials Research Unit University of Leeds, Leeds, UK (Prof LD Sharples)

Correspondence to: Prof Linda Sharples, Clinical Trials Research Unit, University of Leeds, Leeds LS2 9JT, UK randomised studies examining VAT-PP suggest that VAT-PP improved symptom control compared with EEP11 and possibly increased survival compared with biop 2 alone. ${ }^{12}$ The standard approach to control pleural effusion in patients with malignant pleural mesothelioma is talc pleurodesis, either via a chest tube or, more recently, by thoracoscopy.

The MesoVATS trial was designed to establish whether VAT-PP improves survival in patients with pleural effusion secondary to malignant pleural mesothelioma when compared with talc pleurodesis, to provide a full economic analysis of these treatments, and to compare symptom control and quality-of-life outcomes.

\section{Methods}

Study design and participants

MesoVATS was an open-label, parallel-group, multicentre, randomised, controlled trial that recruited 
patients from 12 secondary or tertiary care hospitals in the UK. Eligible patients were those aged older than 18 years with confirmed (any subtype) or suspected (working diagnosis) malignant pleural mesothelioma who had a pleural effusion. Participants had to be fit enough to undergo VAT-PP and provide informed consent. Exclusion criteria were previous attempted pleurodesis and previous primary treatment for mesothelioma. Patients with previous malignancy were eligible if they were no longer receiving anticancer 1 treatment and had a confirmed diagnosis of malignant pleural mesothelioma. Those with a history of malignancy but with only suspected mesothelioma were excluded because the relation of the pleural disease and effusion to the original cancer would have been 1 uncertain. Patients with suspected malignant pleural mesothelioma who were found to have non-malignant disease or non-mesothelioma malignancy after randomisation were excluded.

The trial was coordinated by Papworth Hospital 2 Clinical Trials Unit (Cambridge, UK) and approved by Huntingdon Research Ethics Committee. After sterile talc was re-designated as a medicinal product in April, 2011, the trial was registered with the Medicines and Healthcare Products Regulatory Agency (MHRA) in 2011.

\section{Randomisation}

Patients were recruited by a local principal investigator and were randomised in a 1:1 ratio to either VAT-PP or talc pleurodesis by a computerised random-number See Online for appendix generator in blocks of ten (appendix) via a telephone randomisation line at Papworth Hospital, operated by staff who were independent of the study. Randomisation was stratified according to risk (high vs low) using the European Organisation for Research and Treatment of Cancer (EORTC) prognostic score. ${ }^{13}$ Patients were defined as high risk if they met three or more of the following criteria: white blood cell count of greater than $8.3 \times 10^{9} / \mathrm{L}$; non-epithelioid tumour type (or unknown cell type at randomisation); male sex; or Eastern Cooperative Oncology Group (ECOG) performance status of 1 or higher. The trial was open label, with patients, clinicians, and researchers all aware of the treatment allocation. The trial database was held at Papworth Hospital NHS Foundation Trust.

\section{Procedures}

A detailed overview of the VAT-PP and talc pleurodesis techniques is described in the appendix. To ensure 5 uniformity of approach, all surgeons discussed the VAT-PP procedure, and from Jan 13, 2009, they also recorded tumour extent and lung re-expansion before and after pleurectomy (appendix). At study outset, talc pleurodesis was done using talc slurry via an intercostal 5 chest drain. From November, 2008, the protocol changed to allow talc pleurodesis by thoracoscopic poudrage.
Patients with confirmed mesothelioma at time of randomisation underwent either VAT-PP or talc pleurodesis (appendix). For patients with suspected (working diagnosis) mesothelioma randomly assigned to VAT-PP, two approaches were permitted: either diagnosis was confirmed by thoracoscopy before VAT-PP or VAT-PP was done directly. In the talc pleurodesis group, diagnosis could either be confirmed by thoracoscopic biopsy followed by subsequent talc instillation, or thoracoscopy with biopsy and talc poudrage were done as a single procedure (appendix)

Staging was determined by two radiologists independently using the International Mesothelioma Interest Group (IMIG) tumour stage and was based on the CT done before randomisation. ${ }^{14}$ Differences were resolved by consensus.

Adverse events were documented and assessed by the local investigator for seriousness, severity, and causality, and were recorded on the case report forms at each follow-up visit.

After completion of the study treatment, ongoing management was at the discretion of the managing clinician. No restrictions were placed on the subsequent use of chemotherapy, radiotherapy, or other palliative control measures.

For the economic analysis, all data on resource use was patient-level and obtained at baseline and at 1, 3, 6, and 12 months on a bespoke data collection form (available on request). We multiplied resource use by costs and prices obtained from national sources (appendix). At baseline, data for type of procedure, days on a ward, days in intensive care, and complications were obtained by research nurses using information from hospital records. Follow-up information on hospital bed use, use of primary care services (eg, family doctor, practice nurse), radiotherapy, chemotherapy, hospice care, and diagnostic tests was obtained by researchers during patient interviews at $1,3,6$, and 12 months. Some aspects were confirmed by hospital records-eg, dates of radiotherapy and number of chest radiographs. Information about consultations in primary care was patient-reported.

\section{Outcomes}

The primary outcome was overall survival at 1 year after randomisation. At the end of the study, survival status for all patients was confirmed using the UK Office for National Statistics mortality register. Survival times were censored on June 17, 2013. Secondary outcomes were: presence or absence of apparent pleural effusion as assessed by the reporting radiologist on chest radiograph; quality of life measured using the EuroQol EQ-5D, ${ }^{15}$ EORTC QLQ-C30 general cancer, and EORTC QLQ-LC13 lung cancer questionnaires, ${ }^{16}$ lung function and exercise tolerance measured using spirometry and the shuttle walk test, respectively; complications; and cost to the health service measured by resource use retrieved from patient interviews and hospital records. For patients who 
received their allocated treatment, each measure was 1 assessed at randomisation (baseline) and 1, 3, 6, and 12 months after treatment. For patients who did not undergo their allocated treatment, follow-up visits occurred at 1, 3, 6, and 12 months after randomisation. Because of operational reasons, the shuttle walk test was only available at five of the 12 centres, and therefore was only undertaken by a subset of patients.

\section{Statistical analysis}

On the basis of previous scientific literature ${ }^{17}$ and a local audit, ${ }^{12}$ we estimated 1-year overall survival to be $37 \%$ with talc pleurodesis and 59\% with VAT-PP. To show this difference we needed 90 patients per group $(5 \%$ two-sided significance) to give $80 \%$ power. Allowing for post- 15 randomisation exclusions, we planned to randomly assign 196 patients.

We analysed the primary endpoint in the intention-totreat population of patients with malignant pleural mesothelioma. We estimated 1-year overall survival with 20 the Kaplan-Meier method, stratified for risk status, and compared differences between groups with a log-rank test. We used Cox proportional hazards models, stratified for risk group, to estimate relative risk of death in the 12 months after randomisation. We compared length of 25 hospital stay using the Wilcoxon rank sum test, and complication rates, including persistent pleural effusion rate, with Pearson's $\chi^{2}$ test. Because of the attrition rate in our study sample (due to death), the number of patients available for secondary outcomes decreased 30 during follow-up. Therefore, we compared all repeated measures at each timepoint separately. We assessed overall survival, EQ-5D score, and quality-adjusted survival in high-risk and low-risk subgroups separately in the only preplanned subgroup analysis.

Initially, we analysed secondary outcomes separately at each timepoint in only patients who had completed an assessment. We did several sensitivity analyses for comparisons of EQ-5D scores, quality-adjusted survival outcomes, and costs using a missing-at-random 40 assumption conditional on treatment group, risk group, and either survival status at each follow-up time or follow-up time in the study to death or censoring, and final survival status. For all other secondary outcomes, we restricted analysis to patients who completed each 45 test.

The economic analysis estimated the costs and costeffectiveness of VAT-PP versus talc pleurodesis up to 12 months after randomisation from a National Health Service (NHS) perspective. Resources were valued using 50 NHS reference costs (Department of Health, 2011), unit costs of health and social care, ${ }^{18}$ published literature, and the Papworth Hospital finance department. Costs are expressed in 2011-12 pounds sterling, inflated when necessary. ${ }^{18}$ We converted the EQ-5D, valued using the 55 UK social tariff, ${ }^{19}$ to quality-adjusted life-years (QALYs) using the area under the curve method. ${ }^{20}$
Descriptive analyses show mean total costs by treatment group and differences between treatment group by category of cost for the original procedure and follow-up. We used non-parametric bootstrapping to estimate differences in mean costs with $95 \%$ CIs and incremental cost-effectiveness ratios. We represented uncertainty in cost-effectiveness acceptability curves and

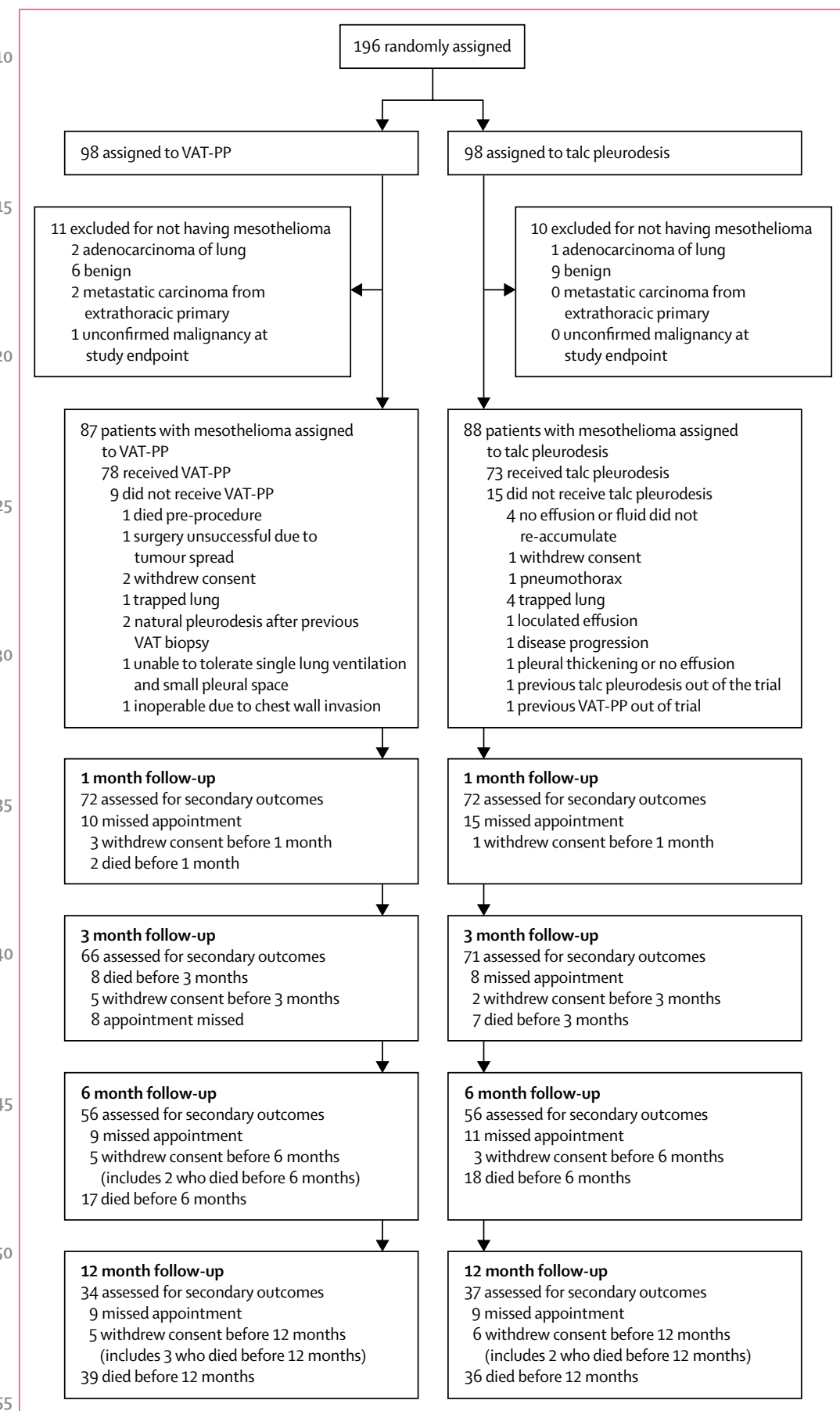

Figure 1: Trial profile

VAT-PP=video-assisted thoracoscopic partial pleurectomy. 
incremental net benefits for VAT-PP versus talc 1 to be related to interventions, and the second half of the pleurodesis. Deterministic sensitivity analysis explored high-risk and low-risk subgroups, complications judged

\begin{tabular}{|c|c|c|}
\hline & VAT-PP $(n=87)$ & Talc pleurodesis $(n=88)$ \\
\hline Age, years & $69.5(7 \cdot 5)$ & $69 \cdot 4(7 \cdot 3)$ \\
\hline \multicolumn{3}{|l|}{ Sex } \\
\hline Men & $75(86 \%)$ & $76(86 \%)$ \\
\hline Women & $12(14 \%)$ & $12(14 \%)$ \\
\hline \multicolumn{3}{|l|}{ EORTC risk status } \\
\hline High & $38(44 \%)$ & $47(53 \%)$ \\
\hline Low & $49(56 \%)$ & $41(47 \%)$ \\
\hline $\mathrm{FEV}_{1} \mathrm{~L}^{*}$ & $1.60(0.62)$ & $1.67(0.56)$ \\
\hline $\mathrm{FEV}_{1}, \%$ predicted $^{*}$ & $57 \cdot 0(18 \cdot 2)$ & $59 \cdot 3(17 \cdot 6)$ \\
\hline FVC, L† & $2 \cdot 21(0.82)$ & $2 \cdot 35(0.75)$ \\
\hline FVC, \% predicted $\dagger$ & $60 \cdot 8(17 \cdot 8)$ & $64 \cdot 2(18 \cdot 3)$ \\
\hline $\mathrm{BMI}, \mathrm{kg} / \mathrm{m}^{2} \ddagger$ & $26 \cdot 6(3.9)$ & $27 \cdot 2(4 \cdot 4)$ \\
\hline Shuttle walk test, $\mathrm{m} \S$ & $405 \cdot 7(154 \cdot 3)$ & $397 \cdot 6(163 \cdot 3)$ \\
\hline \multicolumn{3}{|l|}{ Tumour type } \\
\hline Epithelioid & $73(84 \%)$ & $73(83 \%)$ \\
\hline Sarcomatoid & $10(11 \%)$ & $7(8 \%)$ \\
\hline Biphasic & $4(5 \%)$ & $8(9 \%)$ \\
\hline \multicolumn{3}{|l|}{ IMIG tumour stage } \\
\hline IA & $2(3 \%)$ & $1(1 \%)$ \\
\hline IB & $10(13 \%)$ & $7(9 \%)$ \\
\hline II & $6(8 \%)$ & $9(11 \%)$ \\
\hline III & $38(49 \%)$ & $34(43 \%)$ \\
\hline IV & $21(27 \%)$ & $28(35 \%)$ \\
\hline Missing & $10(11 \%)$ & $9(10 \%)$ \\
\hline \multicolumn{3}{|c|}{ ECOG performance status } \\
\hline 0 & $20(24 \%)$ & $16(19 \%)$ \\
\hline 1 & $50(60 \%)$ & $53(62 \%)$ \\
\hline 2 & $11(13 \%)$ & $14(16 \%)$ \\
\hline 3 & $3(4 \%)$ & $2(2 \%)$ \\
\hline Missing & $3(3 \%)$ & $3(3 \%)$ \\
\hline \multicolumn{3}{|l|}{ Smoking } \\
\hline Current smoker & $5(6 \%)$ & $4(5 \%)$ \\
\hline Ex-smoker & $52(61 \%)$ & $45(52 \%)$ \\
\hline Never smoked & $28(33 \%)$ & $38(44 \%)$ \\
\hline Missing & $2(2 \%)$ & $1(1 \%)$ \\
\hline \multicolumn{3}{|l|}{ Asbestos exposure } \\
\hline Yes & $64(74 \%)$ & $66(76 \%)$ \\
\hline No & $4(5 \%)$ & $1(1 \%)$ \\
\hline Not known & $18(21 \%)$ & $20(23 \%)$ \\
\hline Missing & $1(1 \%)$ & $1(1 \%)$ \\
\hline \multicolumn{3}{|l|}{ Dyspnoea } \\
\hline Yes & $67(78 \%)$ & $68(78 \%)$ \\
\hline No & $19(22 \%)$ & $19(22 \%)$ \\
\hline Missing & $1(1 \%)$ & $1(1 \%)$ \\
\hline
\end{tabular}

Data are mean (SD) or number (\%). VAT-PP=video-assisted thoracoscopic partial pleurectomy. EORTC=European Organisation for Research and Treatment of $\mathrm{Cancer}_{\text {. }} \mathrm{FEV}_{1}=$ forced expiratory volume in $1 \mathrm{~s}$. FVC=forced vital capacity. $\mathrm{BMI}=$ body-mass index. IMIG=International Mesothelioma Interest Group. ECOG=Eastern Cooperative Oncology Group ${ }^{*} n=76$ in talc pleurodesis group; $n=79$ in VAT-PP group. $n n=75$ in talc pleurodesis group; $n=79$ in VAT-PP group. $\neq n=74$ in talc pleurodesis group; $n=74$ in VAT-PP group. $\$ n=49$ in talc pleurodesis group; $n=53$ in VAT-PP group.

Table 1: Baseline demographic and clinical characteristics in randomly assigned patients with mesothelioma trial only (any patients randomly assigned after June 1 , 2009) to account for changing clinical practice over time. We finalised these subgroup analyses after trial 5 completion, and they were exploratory.

This study is registered with an International Standard Randomised Controlled Trial Number, 34321019; the European Clinical Trials Database (EudraCT), number 2011-001121-24; and with ClinicalTrials.gov, number NCT00821860

\section{Role of the funding source}

The funder of the study had no role in study design, data collection, data interpretation, or writing of the report. 15 All authors had full access to all the data in the study and had final responsibility for the decision to submit for publication.

\section{Results}

20 As planned, we recruited 196 patients between Oct 24, 2003, and Jan 24, 2012 (figure 1), and followed them up until Jan 31, 2013, with a further survival update on June 17, 2013. At randomisation, 120 (61\%) of 196 patients had confirmed malignant pleural mesothelioma and 76 (39\%) 25 had suspected (working diagnosis) malignant pleural mesothelioma (appendix). $11(11 \%)$ of 98 patients assigned to VAT-PP and 10 (10\%) of 98 patients assigned to talc pleurodesis with suspected malignant pleural mesothelioma were subsequently found to have benign o disease $(n=15)$, adenocarcinoma of the lung $(n=3)$, metastatic disease from an extrathoracic primary cancer $(n=2)$, or unconfirmed malignant pleural mesothelioma at study endpoint $(n=1)$, and were excluded (figure 1$)$. We analysed the remaining 87 patients in the VAT-PP group 35 and 88 patients in the talc pleurodesis group in the intention-to-treat analyses.

Within 12 months of randomisation, 42 (48\%) of 87 patients in the VAT-PP group had died compared with $38(43 \%)$ of 88 in the talc pleurodesis group; $4014(16 \%)$ patients in the VAT-PP group and $15(17 \%)$ in the talc pleurodesis group either withdrew from the study or did not attend the final appointment (figure 1). Thus, at 12 months secondary outcomes were available for $34(39 \%)$ of 87 patients in the VAT-PP group and $4537(42 \%)$ of 88 patients in the talc pleurodesis group, although some secondary outcomes were available for $72(83 \%)$ of 87 patients in the VAT-PP group and $72(82 \%)$ of 88 patients in the talc pleurodesis group. One patient withdrew following an MHRA decision, 50 during the trial, to reclassify talc as a medicinal product, and the trial was suspended until MHRA registration. Other reasons for withdrawal from the trial are given in the appendix.

Baseline characteristics were similar between the two 55 groups (table 1). Mean age was 69 years (SD 7.4) in all 175 randomly assigned patients with mesothelioma, and 151 (86\%) were men. Most patients had epithelioid 
tumours (table 1). Three patients had a history of cancer (one had breast cancer and another had bladder cancer in the VAT-PP group, and one had prostate cancer in the talc pleurodesis group). ECOG performance status was 0 or 1 for 139 (82\%) of the 169 patients for whom data were available, and IMIG tumour stage was IA in three $(2 \%)$ of 156 patients with available data, IB in $17(11 \%)$ patients, II in $15(10 \%)$ patients, III in $72(46 \%)$ patients, and IV in $49(31 \%)$ patients. Exposure to asbestos was reported by 130 (75\%) of 173 patients with available data, and breathlessness was reported by $135(78 \%)$ of 173 , with a percent-predicted mean forced expiratory volume in $1 \mathrm{~s}\left(\mathrm{FEV}_{1}\right)$ of $58 \cdot 1 \%(\mathrm{SD} 17 \cdot 8)$.

Nine $(10 \%)$ of 87 patients in the VAT-PP group did not undergo their assigned procedure, compared with $15(17 \%)$ of 88 patients in the talc pleurodesis group (figure 1). Talc pleurodesis commenced a median of 9 days (IQR 6-15) after randomisation in 73 patients, with 35 (48\%) receiving talc slurry and 34 (47\%) undergoing pleurodesis via thoracoscopy (data for the 20 remaining four patients are unavailable). VAT-PP was completed a median of 14 days (IQR 8-21) after randomisation.

Overall survival at 6 months was estimated to be $78 \%$ (95\% CI 68-85) in the VAT-PP group and 80\% (70-87) in 25 the talc pleurodesis group, and at 1 year was estimated to be 52\% (41-62) and 57\% (46-66), respectively (hazard ratio [HR] 1.04 [95\% CI 0.76-1.42]; $\mathrm{p}=0 \cdot 81$; figure $2 \mathrm{~A}$ ). Median overall survival was $13 \cdot 1$ months (IQR 7·3-20 3) in the VAT-PP group and $13 \cdot 5$ months $(7 \cdot 3-21 \cdot 1)$ in the 30 talc pleurodesis group. Overall survival at 6 months was estimated to be $66 \%$ (95\% CI $48-78$ ) in patients in the VAT-PP group who were at high risk according to the EORTC prognostic score and 74\% (59-85) in patients in the talc pleurodesis group who were at high risk; for 3 patients who were at low risk, overall survival at 6 months was $88 \%$ (75-94) and 85\% (70-93), respectively (figure 2B). At 1 year, overall survival was 37\% (95\% CI 22-52) in high-risk patients in the VAT-PP group and $53 \%(38-66)$ in high-risk patients in the talc pleurodesis 40 group, and 63\% (48-75) and 61\% (44-74) in the low-risk group for VAT-PP and talc pleurodesis, respectively (figure 2B). Overall survival rates were not significantly different between the treatment groups (stratified logrank test $\mathrm{p}=0 \cdot 51$ ). The HR of death for the VAT-PP group 45 relative to the talc pleurodesis group, stratified by EORTC prognostic risk, was $1 \cdot 11$ (95\% CI 0.81-1.53; p=0 51).

Pleural effusion was reported as apparently having resolved in $25(37 \%)$ of 68 surviving patients in the talc pleurodesis group at 1 month, in $37(60 \%)$ of 62 at 50 3 months, in 31 (57\%) of 54 at 6 months, and in 27 (77\%) of 35 at 12 months. Corresponding numbers in the VATPP group were 41 (59\%) of 69 patients at 1 month, $36(60 \%)$ of 60 at 3 months, 41 (77\%) of 53 at 6 months, and $23(70 \%)$ of 33 at 12 months. The proportion of 55 patients with resolved pleural effusion was significantly higher in the VAT-PP group than in the talc pleurodesis group at 1 month $(\mathrm{p}=0.008 \mathrm{X})$ and 6 months $(\mathrm{p}=0.03 \mathrm{X})$, but not at 3 months $(\mathrm{p}=0 \cdot 97)$ or 12 months $(\mathrm{p}=0 \cdot 49)$.

Of the 175 randomly assigned patients with malignant pleural mesothelioma, four patients withdrew within 1 month, of whom two did not provide baseline data and two did; a further two patients did not complete baseline quality-of-life questionnaires, and three had one or more missing baseline EQ-5D items, leaving 83 patients in the VAT-PP group and 85 patients in the talc pleurodesis group with baseline EQ-5D data. The pattern of missing data during follow-up, which was more frequent just before death, is shown in the appendix. EQ-5D results are shown in figure 3 and the appendix. Patients in the VATPP group had slightly worse EQ-5D utility index scores at 1 month than patients in the talc pleurodesis group (mean difference -0.06 [95\% CI -0.13 to 0.004$]$; $p=0.07 \mathrm{X}$ ), slightly better EQ-5D scores at 3 months (mean difference

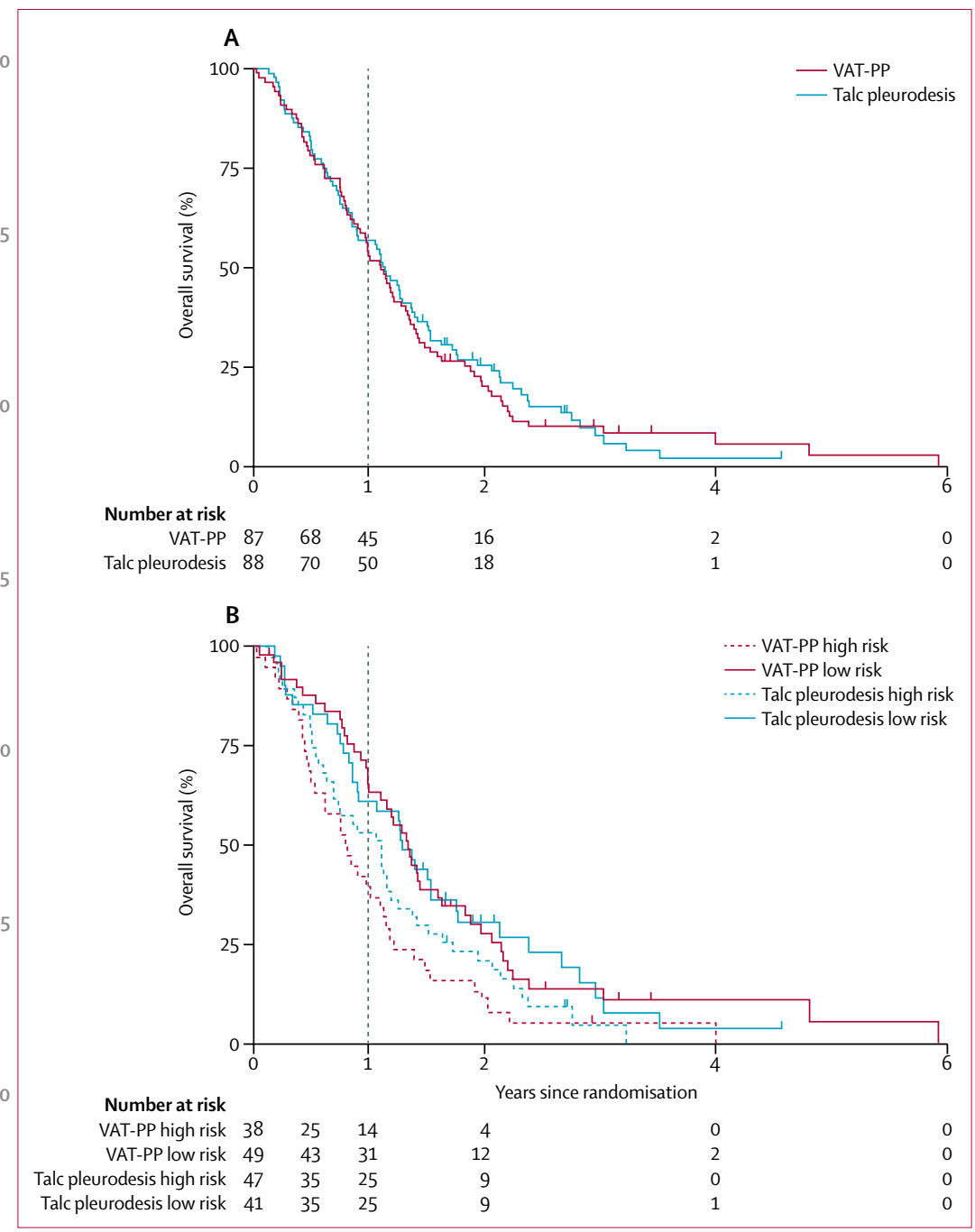

Figure 2: Kaplan-Meier curves of overall survival, in all patients and by risk group Overall survival in all randomly assigned patients with mesothelioma, per treatment group (A) and in high-risk and low-risk patients, per treatment group (B). The vertical line crosses the $x$-axis at 1 year (primary endpoint). VAT-PP=video-assisted thoracoscopic partial pleurectomy. 
$0 \cdot 04[-0 \cdot 03$ to $0 \cdot 12] ; p=0 \cdot 27)$, and significantly better EQ$5 \mathrm{D}$ scores at 6 months (mean difference $0.08[0.003$ to $0 \cdot 16$; $\mathrm{p}=0.042$ ) and $1 \bigcirc$ honths (mean difference 0.19 [0.05 to 0.32$] ; p=0.000 \mathrm{X})$. The mean number of QALYs in the VAT-PP group was 0.511 (95\% CI 0 446-0 - 577) compared with $0.476(0.418-0 \cdot 534)$ in the talc pleurodesis group (mean difference 0.035 [-0.051 to $0 \cdot 122] ; \mathrm{p}=0.42$; table 2 ). Sensitivity analysis using a range of assumptions about missing data resulted in very similar estimates (appendix) and estimates of mean QALYs from all analyses were consistent.

Results of the analysis of some of the EORTC subscales (eg, global health, physical functioning, and role functioning) were similar to the results from the EQ-5D analysis (poorer function at 1 month and better function at 3, 6, and 12 months with VAT-PP than with talc pleurodesis), but results were not consistent between scales, nor were the differences noted between groups consistently significant (appendix).

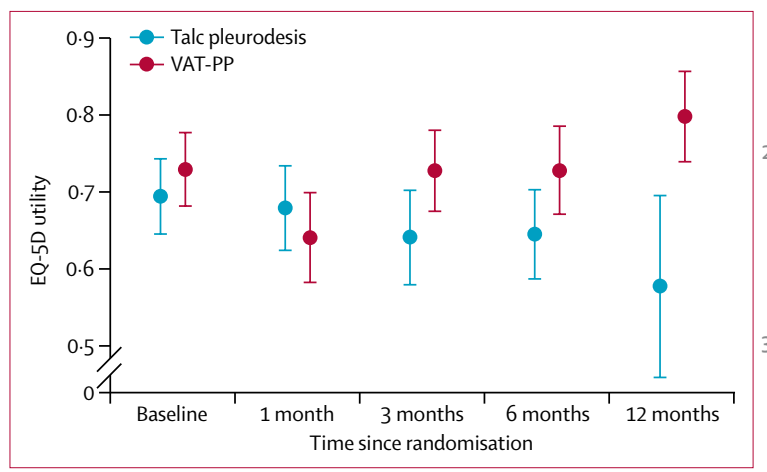

Figure 3: EQ-5D score during the $\mathbf{1 2}$ months after randomisation Data are from patients who completed the ED-5D questionnaire at each timepoint (at baseline, 83 in VAT-PP group and 85 in talc pleurodesis group: 1 month, $n=71$ and $n=70 ; 3$ months, $n=63$ and $n=71 ; 6$ months, $n=55$ and $n=56$; and 12 months, $n=33$ and $n=36$ ). Datapoints show mean ED-5D utility (indexbased value) and bars show $95 \% \mathrm{CI}$. VAT-PP=video-assisted thoracoscopic partial pleurectomy.

Patients in the VAT-PP group had higher mean $\mathrm{FEV}_{1}$ at 1 , 3 , and 12 months after treatment than did patients in the talc pleurodesis group, despite lower baseline function, but differences were not statistically significant (appendix). Forced vital capacity did not differ between groups (appendix). Patients in the talc pleurodesis group recorded a greater mean distance in the shuttle test, and the difference was significant at 12 months (difference $103.5 \mathrm{~m}$ [95\% CI 37.1-169.9]; $\mathrm{p}=0 \cdot 003 \mathrm{X}$ ), although this analysis was based on data from only 36 patients because the test was only done in five of the 12 participating centres.

We did not note any significant interactions between risk group and treatment group, but high-risk patients had significantly worse overall survival than low-risk patients (HR 1.64 [95\% CI 1.19-2.5] $\mathrm{p}=0.002 \mathrm{X}$ ) and although some evidence suggested that high-risk patients who underwent VAT-PP had poorer overall survival than high-risk patients who underwent talc pleurodesis, the difference in overall survival between groups was not 20 significant (HR 1.29 [95\% CI 0.83-2.00]; $\mathrm{p}=0 \cdot 25$ ). The difference in mean EQ-5D score at 12 months in favour of patients with VAT-PP was significant for low-risk patients (mean difference 0.13 [95\% CI $0 \cdot 02-0 \cdot 24]$ $\mathrm{p}=0 \cdot 022$ ) and was large, but not significant, for high-risk 25 patients (mean difference $0 \cdot 32$ [ $-0 \cdot 04$ to $0 \cdot 67] ; \mathrm{p}=0 \cdot 08 \mathrm{X}$ ).

The mean cost of VAT-PP treatment and follow-up care for 12 months was about $£ 3800$ more than the cost of talc pleurodesis (appendix). Differences were mainly attributable to the initial procedure and increased length o of stay, followed by the cost of admissions during followup and treatment of surgical complications. The cost of gaining an additional QALY from VAT-PP is $£ 109000$ (table 3). A potential willingness-to-pay per QALY of $£ 30000$ is associated with a $5 \cdot 4 \%$ chance that VAT-PP

35 was cost-effective relative to talc pleurodesis, and a willingness to pay per QALY of $£ 50000$ is associated with a $20 \%$ chance (figure 4 ).

Deterministic sensitivity analysis (table 3 ) shows that high-risk patients in the VAT-PP group had fewer QALYs 40

\begin{tabular}{|c|c|c|c|}
\hline & All patients & High-risk group & Low-risk group \\
\hline \multicolumn{4}{|l|}{ Total cost } \\
\hline Talc pleurodesis & $10436(14 \cdot 4)$ & $11408(19 \cdot 0)$ & $9359(21 \cdot 6)$ \\
\hline VAT-PP & $14252(14 \cdot 4)$ & $13688(19 \cdot 1)$ & $14663(21 \cdot 7)$ \\
\hline \multicolumn{4}{|l|}{ QALYs } \\
\hline Talc pleurodesis & $0.476(0.0005)$ & $0.409(0.0007)$ & $0.555(0.0007)$ \\
\hline VAT-PP & $0.511(0.0006)$ & $0.383(0.0007)$ & $0.607(0.0009)$ \\
\hline Difference in cost & $3816(20 \cdot 7)$ & $2274(21 \cdot 7)$ & $5305(19 \cdot 1)$ \\
\hline Difference in QALYs & $0.035(0.0008)$ & $-0.026(0.00085)$ & $0.050(0.0007)$ \\
\hline Incremental cost per QALY gained & 109028 & VAT-PP dominated by TP* & 105119 \\
\hline Incremental net benefit at $£ 30000$ per QALY & -2766 & -3054 & -3805 \\
\hline
\end{tabular}

Data are mean (SE) unless otherwise specified. Costs are presented in 2011 pound sterling (GBPE). This analysis uses bootstrapped estimates, not the imputed dataset (used in appendix). VAT-PP=video-assisted thoracoscopic partial pleurectomy. TP=talc pleurodesis. QALY=quality-adjusted life-year. *An intervention (ie, VAT-PP) is dominated when it is less effective and more costly than the comparator (ie, talc pleurodesis).

Table 2: Comparison of costs and QALYs gained from VAT-PP versus talc pleurodesis 
and were more expensive to treat than were high-risk patients who received talc pleurodesis, whereas the lowrisk group had an additional cost of $£ 105119$ per QALY gained for the VAT-PP group compared with the talc pleurodesis group. Using results from the second half of 5 the trial only (after June 1, 2009; n=98), VAT-PP had a greater effect, with the incremental cost-effectiveness ratio falling to $£ 68600$ per QALY gained (appendix).

Of the 78 patients who completed VAT-PP, respiratory complications were reported in 19 (24\%) patients, 1 cardiac complications in five (6\%), and other (surgical) complications in 24 (31\%). Of the 73 patients who completed talc pleurodesis, 11 (15\%) had respiratory complications $(\mathrm{p}=0.22$ for difference between treatment groups), three $(4 \%)$ had cardiac complications $(\mathrm{p}=0 \cdot 84), 15$ and ten (14\%) had surgical complications $(\mathrm{p}=0 \cdot 019)$. Some patients had more than one type of complication. Air leak beyond 2 days was significantly more common after VAT-PP than after talc pleurodesis, occurring in $19(24 \%)$ of 78 patients in the VAT-PP group versus 2 four $(5 \%)$ of 73 patients in the talc pleurodesis group $(\mathrm{p}=0.001 \mathrm{X}) \mathrm{P}$ rsistent air leak ( $>10$ days) was more frequent in 2 VAT-PP group, but not significantly so (five [6\%] vs one [1\%]; $\mathrm{p}=0 \cdot 21$ ). 21 serious adverse events were related to the trial medication or procedure; 13 (in 2 11 patients) were in the VAT-PP group and eight (in seven patients) were in the talc pleurodesis group $(\mathrm{p}=0 \cdot 35$; table 3). Full details of treatment complications are presented in the appendix. Median hospital stay after the procedure was 7 days (IQR 5-11) for the VAT-PP group 3 and 3 days (IQR 2-5) for the talc pleurodesis group $(p<0 \cdot 0001)$. A similar proportion of patients in each group had chemotherapy after their study procedure (28 [32\%] of 87 in the VAT-PP group vs 25 [28\%] of 88 in the talc pleurodesis group), with a mean of 35 4.4 cycles (SD $2 \cdot 4$ ) and $4 \cdot 5$ cycles $(3 \cdot 2)$, respectively.

\section{Discussion}

To our knowledge, our study is the first randomised controlled trial to compare VAT-PP and talc pleurodesis 40 in patients with suspected or confirmed malignant pleural mesothelioma. Our results showed that overall survival was not improved by VAT-PP, and that this approach resulted in more complications, longer hospital stays, and was more expensive than was talc pleurodesis.

A study in a similar population of patients-the Mesothelioma and Radical Surgery (MARS) trial ${ }^{7}-$ compared extrapleural pneumonectomy (EPP) plus postoperative hemithoracic irradiation with no EPP in patients with mesothelioma who had received induction 5 platinum-based chemotherapy. Although this feasibility study showed that recruitment and randomisation to EPP within the context of trimodality therapy was achievable, the adjusted HR in favour of not having EPP was $2 \cdot 75(95 \%$ CI $1 \cdot 21-6 \cdot 26 ; \mathrm{p}=0 \cdot 016)$. In view of the 5 high morbidity associated with EPP, a larger study was not undertaken.

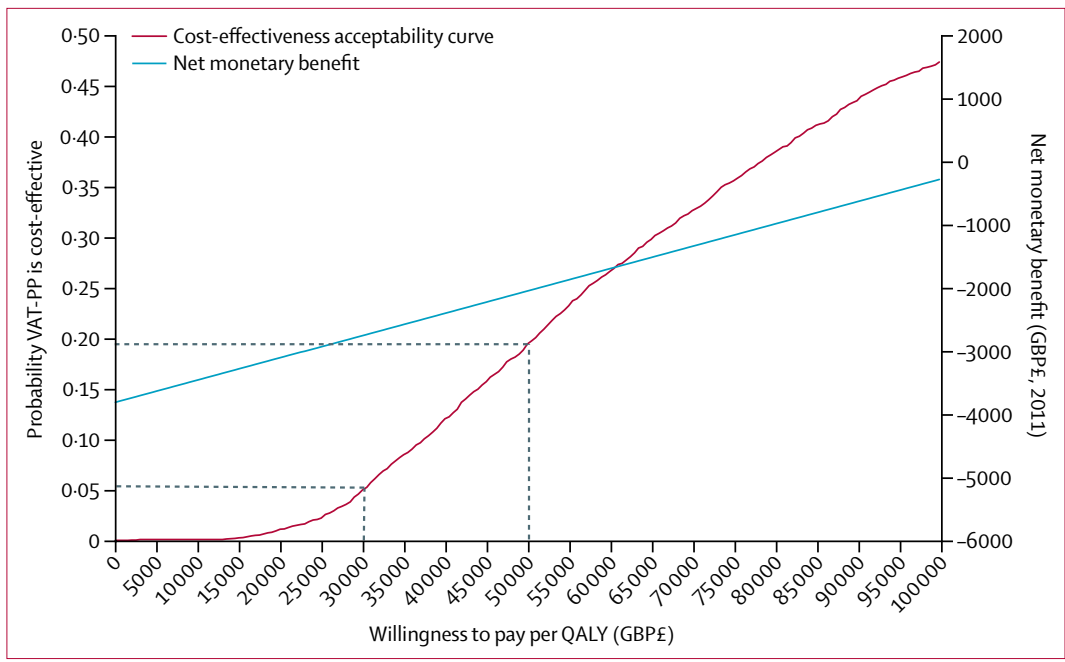

Figure 4: Cost-effectiveness and net monetary benefit of VAT-PP

$\mathrm{QALY}=$ quality-adjusted life-year. VAT-PP=video-assisted thoracoscopic partial pleurectomy.

\begin{tabular}{|c|c|c|}
\hline & $\begin{array}{l}\text { VAT-PP } \\
(n=78)\end{array}$ & $\begin{array}{l}\text { Talc pleurodesis } \\
(n=73)\end{array}$ \\
\hline Serious adverse events & $13(17 \%)$ & $8(11 \%)$ \\
\hline Death & $1(1 \%)$ & 0 \\
\hline \multicolumn{3}{|l|}{ Extended hospital stay } \\
\hline Renal failure & $1(1 \%)$ & 0 \\
\hline Surgical emphysema & $1(1 \%)$ & 0 \\
\hline Repeat VAT-PP and subsequent CVA & $2(3 \%)$ & 0 \\
\hline Fever and dyspnoea & 0 & $1(1 \%)$ \\
\hline \multicolumn{3}{|l|}{ Re-admissions } \\
\hline Leaking drain & $3(4 \%)$ & 0 \\
\hline Persistent pleura effusion & $1(1 \%)$ & $3(4 \%)$ \\
\hline Hydropneumothorax & $1(1 \%)$ & 0 \\
\hline Empyema & $1(1 \%)$ & $1(1 \%)$ \\
\hline Infected drain site & 0 & $2(3 \%)$ \\
\hline Thoracotomy with decortication & 0 & $1(1 \%)$ \\
\hline Symptom control & $1(1 \%)$ & 0 \\
\hline \multicolumn{3}{|l|}{ Other } \\
\hline Awareness under anaesthesia & $1(1 \%)$ & 0 \\
\hline \multicolumn{3}{|c|}{$\begin{array}{l}\text { Data are number of events (\% of patients). VAT-PP=video-assisted thoracoscopic } \\
\text { partial pleurectomy. CVA=cerebrovascular accident. }\end{array}$} \\
\hline \multicolumn{3}{|c|}{$\begin{array}{l}\text { Table 3: Deaths and serious adverse events possibly or probably related } \\
\text { to treatment }\end{array}$} \\
\hline
\end{tabular}

In recognition that many patients with mesothelioma present with advanced disease and comorbidities, MesoVATS assessed VAT-PP—a less arduous surgery than EPP. However, we found that this approach does not confer a survival advantage over talc pleurodesis and resulted in longer hospital stays. Median overall survival was 13.1 months in the VAT-PP group and 13.5 months and in the talc pleurodesis group (compared with 14.4 months reported in the EPP group of MARS ${ }^{7}$ ) and was at the upper end of the range of 7-14 months reported in the systematic review of 14 observational studies and 
case series of partial pleurectomy. ${ }^{21} 1$-year overall survival in the VAT-PP group in our study $(52 \%)$ was similar to our pre-trial estimate of $59 \%$; however, our pre-trial estimate for the talc pleurodesis group of $37 \%$, which was derived from audit data, was very different to the actual result $(57 \%)$, and was probably based on less carefully selected patients than those put forward for VAT-PP. This result shows the bias inherent in many non-randomised comparisons.

So far, few studies into partial pleurectomy for 1 malignant pleural mesothelioma have been published (panel). Since most patients with malignant pleural mesothelioma present with advanced disease, the findings from MesoVATS could be generalised, because $78 \%$ of patients with a final diagnosis of malignant pleural mesothelioma were at IMIG stage III/IV and most had an ECOG performance status of 1 . These results contrast with the more highly selected patients in the MARS trial who were predominantly IMIG stage II and had an ECOG performance status of 0 or 1. The

\section{Panel: Research in context}

\section{Systematic review}

We searched Medline, Embase, Cumulative Index to Nursing and Allied Health Literature, and the Cochrane Library for articles comparing talc pleurodesis with video-assisted thoracoscopic partial pleurectomy (VAT-PP) in patients with mesothelioma, using the keywords "mesothelioma", "pleurect", "thoracic surgery", and "talc pleurod". We screened the relevance of studies by assessment of titles, keywords, abstracts, and full texts. We used a Critical Appraisal Skills Programme checklist to assess the quality of the evidence. We found a few published studies that were similar to our own; however, surgical approaches that aim to remove all macroscopically visible tumour (extrapleural pneumonectomy and extended pleurectomy decortication) are not directly comparable with techniques such as partial pleurectomy, in which the aim is to partially remove parietal or visceral pleura for diagnostic or palliative purposes. ${ }^{6}$ Second, high-level evidence for partial pleurectomy is scarce in this setting. No randomised trials involving partial pleurectomy have been reported. Cao and colleagues ${ }^{21}$ reported a systematic review of safety and efficacy of pleurectomy (extended pleurectomy with decortication and partial pleurectomy) in patients with malignant pleural mesothelioma. Of 14 studies identified as containing patients who had undergone partial pleurectomy (assigned retrospectively on the basis of International Mesothelioma Interest Group or International Association for the Study of Lung Cancer criteria), most were retrospective, none assessed quality of life, and data completeness was variable. Surgical approaches varied between thoracotomy and video-assisted thoracoscopic surgery. Since all studies were non-randomised case series involving selected patients, spanning a wide timeframe (1958-2008), with variable methods and reporting, we found it difficult to draw conclusions other than noting that median survival ranged from 7 to 14 months.

\section{Interpretation}

Our trial is the first, to our knowledge, to compare VAT-PP and talc pleurodesis in a randomised controlled trial. With regard to future clinical practice, our results show that VAT-PP does not offer a survival advantage over talc pleurodesis and is associated with more surgical complications. However, we found some evidence that low-risk patients who are expected to survive at least 6 months might benefit in terms of quality of life from VAT-PP. Future randomised studies of lung sparing (extended) pleurectomy with decortication at thoracotomy should examine whether more extensive pleural resection confers benefit in terms of survival and quality-of-life measures. advanced stage of disease at randomisation in our trial might explain why VAT-PP did not confer a survival advantage over talc pleurodesis; the more limited excision of tumour by VAT-PP than by EPP might have been insufficient to have an effect on overall survival. Future randomised studies examining lung-sparing (extended) pleurectomy with decortication at thoracotomy will establish whether more extensive resection provides a survival benefit with acceptable morbidity.

In the VAT-PP group, EQ-5D-assessed quality of life for surviving patients at 6 and 12 months was significantly better than that in the talc pleurodesis group. From a patient perspective, this finding is important because quality of life is a primary concern in the final months of life. Although the clinical significance of the observed improvements in EQ-5D is unclear since no pre-trial minimally important difference was posited, Pickard and colleagues $^{22}$ suggest that a difference in EQ-5D utility in the range of $0 \cdot 07-0.12$ is important for patients with lung cancer. Differences of this order were achieved at 6 and 12 months in the VAT-PP group compared with the talc pleurodesis group. However, the EQ-5D results were at odds with two cancer-specific quality-of-life questionnaires that did not show consistent improvements in the 25 VAT-PP group. Further studies examining the reasons for the variation in outcomes between the quality-of-life questionnaires in this scenario are required.

Historically, control of pleural effusion has been by talc pleurodesis, either using talc slurry via an intercostal 30 drain, or by talc poudrage at thoracoscopy. During the study, practice shifted from administration of talc slurry via a chest drain towards increasing use of talc poudrage at thoracoscopy in line with contemporary practice (appendix). Previous studies of talc pleurodesis efficacy 35 have shown effusion control rates (partial or complete) of between $60 \%$ and $84 \%$, but these were in heterogeneous populations containing few patients with mesothelioma. ${ }^{23,24}$ Studies of talc pleurodesis efficacy focusing specifically on patients with mesothelioma seem to have 40 lower success rates than studies in heterogeneous populations, probably because extensive visceral disease, common in mesothelioma, mitigates against successful pleurodesis because of failure of visceral and parietal pleura apposition combined with a paucity of normal 45 mesothelial cells. ${ }^{25,26}$ A retrospective study ${ }^{27}$ of 165 patients with malignant pleural mesothelioma who underwent pleurodesis reported that pleural effusion was not controlled in $31 \%$ of patients, with no difference between talc slurry and talc poudrage. At the inception of our trial, 50 the predominant method for assessing pleural effusion was chest radiograph-a less sensitive method than ultrasound, which is now widely used. In some cases, pleural tumour or pleural reaction after pleurodesis or pleurectomy might have been mistaken for a small 55 recurrent pleural effusion. This factor should be taken into account when interpreting the results relating to fluid control. Others have defined failure of pleurodesis 
as the need for further pleural intervention for fluid control. ${ }^{28}$ Data for subsequent pleural interventions were not routinely obtained in our study.

In the economic analysis, VAT-PP cost an additional $£ 3800$ per patient compared with talc pleurodesis. The additional $0 \cdot 035$ QALYs gained per patient (equivalent to 12.5 days of full health) could be bought at a rate of $£ 109000$ per QALY. Due to the prolonged recruitment period and changes in management over time, we estimated cost-effectiveness in only patients recruited in the second half of the trial ( 98 patients randomised after June 1, 2009). For this group, the cost per QALY was $£ 68600$, which is closer to, but in excess of, the highest cost per QALY of a drug $(£ 50000)$ accepted by NICE in previous decisions for end-of-life treatments. ${ }^{29}$ The reason for the improvement in cost-effectiveness is multifactorial. After study midpoint, trial patients undergoing VAT-PP had a slightly lower median hospital stay than did earlier patients (7 days [IQR 5-7] vs 8 days [6-11]) whereas patients with talc pleurodesis had a very similar but slightly higher median hospital stay than did earlier patients (4 days [2-7] vs 3 days [2-5]), resulting from greater use of thoracoscopy and talc poudrage in the second half of the trial (appendix), which is more expensive (talc pleurodesis cost $£ 1129$ in the first half of the trial and $£ 2649$ in the second half). Additionally, we noted some evidence of a greater difference between the groups in EQ-5D score at 6 and 12 months in the second half of the trial, whereas survival remained similar for the two groups (appendix).

Since VAT-PP was both more expensive and less effective than talc pleurodesis in the high-risk subgroup, there seems to be little rationale for this procedure in these patients. However, treatment of low-risk patients with VAT-PP might have some economic justification if the end-of-life willingness-to-pay threshold is raised to $£ 68600$ per QALY or if future studies show that the costs of VAT-PP relative to talc pleurodesis continue to decline further. Further refinement of risk classification systems might also help to identify patients who might benefit most from each treatment.

During the 10 years of study recruitment, several changes in the clinical management of malignant pleural mesothelioma occurred. With regard to staging, MesoVATS used CT, although recent research has shown that although CT remains the first-line investigation method, PET-CT, and in certain circumstances, MRI, might improve the accuracy of staging. ${ }^{30,31}$ NICE guidance $(2008)^{29}$ on cisplatin plus pemetrexed chemotherapy for mesothelioma resulted in more patients in the second 5 half of the study receiving chemotherapy after study treatment. Because similar numbers of patients in each group had post-procedure chemotherapy, we felt the use of chemotherapy in the clinical management of the disease did not unduly bias the results.

The poor prognosis and high morbidity in this group of patients meant that secondary outcomes were available for less than half the population by 12 months, although results were robust to a range of missing data analyses. Because the study was open label with group allocations available to patients, clinicians, and researchers, we cannot exclude bias in secondary outcomes as a result of a patient or clinician having a strong preference for one of the treatments.

In conclusion, VAT-PP had no effect on overall survival, resulted in more complications, longer hospital stay, and was more expensive than talc pleurodesis in patients with pleural effusion due to malignant pleural mesothelioma. However, significant improvement in EQ-5D score at 6 and 12 months in the VAT-PP group suggests that this treatment might have a role in patients expected to survive at least 6 months. Subgroup analysis suggests that patients in the EORTC low-risk prognostic group might benefit most from VAT-PP, and further work in this subgroup might be appropriate.

\section{Contributors}

RCR was the chief investigator for this trial (2009 onwards). AJR was one of the original instigators of the study. RCR, AJR, DAW, JGE, and ASC were involved in recruitment, clinical care, and data collection. The trial was coordinated by RCR and VH, and data analysis was done by LDS, MB, EL, and JAF-R. RCR, LDS, and JAF-R interpreted the data and wrote the first draft of the manuscript, and all authors contributed to subsequent editing. All authors reviewed and approved the final version of the paper. The authors accept full responsibility for the overall content of the paper. Day-to-day running of the trial was undertaken by RCR, $\mathrm{VH}$, and LDS under the guidance of the trial steering committee.

\section{MesoVATS Collaborators}

Paul Beckett (Burton Hospital NHS Foundation Trust); 20 Loci Lang-Lazdunski (Guy's and St Thomas' NHS Foundation Trust); Raja Reddy (Kettering General Hospital, NHS Foundation Trust); Duncan Powrie (Southend University Hospital NHS Foundation Trust); Eric Lim (Royal Brompton and Harefield NHS Foundation Trust); Antonio Martin-Ucar (Nottingham University Hospitals NHS Trust) Ian Morgan (Royal Wolverhampton Hospital NHS Trust);

Nilanjan Chaudhuri (St James University Hospital, Leeds);

Nagmi Qureshi, Angela Tasker, Helen Munday, Claire Matthews, Robert Buttery, Mark Slade, Gerry Slade, Doris Rassl, Erica Lowry, and Jane Elliott (Papworth Hospital NHS Foundation Trust); Claire Laroche (West Suffolk Hospital NHS Foundation Trust); and Robert Winter (Addenbrooke's Hospital NHS Foundation Trust).

\section{Declaration of interests}

RCR has been a member of an advisory board for Lilly UK. JGE has received honoraria from Lilly UK. All others authors declare no competing interests.

\section{Acknowledgments}

Grant funding was obtained from the BUPA Foundation. RCR was 5 funded, in part, by the Cambridge Biomedical Research Centre and the Cambridge Cancer Centre, and LDS was partly funded by the Medical Research Council programme, number U015232027. MB was funded by a National Institute for Health Research Clinical Trials Methodology fellowship. We are grateful to the MesoVATS trial steering committee: Richard Himsworth (Chair), John Cairns, Aman Coonar, John Edwards, Vikki Hughes, Loic Lang-Lazdunski, Eric Lim, Antonio Martin-Ucar, Ian Morgan, Andrew Ritchie, Robert Rintoul, Linda Sharples, Richard Stephens, David Waller, Jane Warwick, and Robert Winter. We also thank the data monitoring committee: Richard Himsworth (Chair), John Cairns, Vikki Hughes, Martin Muers, Linda Sharples, Richard Stephens, and Jane Warwick. We thank all the staff at the participating centres, the referring clinicians, and especially the patients

for their participation. [A: I could not see correspondence from the names highlighted above---please resend 


\section{References}

1 Peto J, Hodgson JT, Matthews FE, Jones JR. Continuing increase in mesothelioma mortality in Britain. Lancet 1995; 345: 535-39.

2 Hodgson JT, McElvenny DM, Darnton AJ, Price MJ, Peto J. The expected burden of mesothelioma mortality in Great Britain from 2002 to 2050. Br J Cancer 2005; 92: 587-93.

3 Peto J, Decarli A, La Vecchia C, Levi F, Negri E. The European mesothelioma epidemic. Br J Cancer 1999; 79: 666-72.

4 Vogelzang NJ, Rusthoven JJ, Symanowski J, et al. Phase III study of pemetrexed in combination with cisplatin versus cisplatin alone in patients with malignant pleural mesothelioma. J Clin Oncol 2003; 21: 2636-44.

5 Bottomley A, Gaafar R, Manegold C, et al, and the EORTC Lung-Cancer Group, and the National Cancer Institute, Canada. Short-term treatment-related symptoms and quality of life: results from an international randomized phase III study of cisplatin with or without raltitrexed in patients with malignant pleural mesothelioma: an EORTC Lung-Cancer Group and National Cancer Institute, Canada, Intergroup Study. J Clin Oncol 2006; 24: 1435-42.

6 Rice D, Rusch V, Pass H, et al, for the International Association for the Study of Lung Cancer International Staging Committee and the International Mesothelioma Interest Group. Recommendations for uniform definitions of surgical techniques for malignant pleural mesothelioma: a consensus report of the International Association for the Study of Lung Cancer International Staging Committee and the International Mesothelioma Interest Group. J Thorac Oncol 2011; 6: 1304-12.

7 Treasure T, Lang-Lazdunski L, Waller D, et al, for the MARS trialists. Extra-pleural pneumonectomy versus no extra-pleural pneumonectomy for patients with malignant pleural mesothelioma: clinical outcomes of the Mesothelioma and Radical Surgery (MARS) randomised feasibility study. Lancet Oncol 2011; 12: 763-72.

8 Flores RM, Pass HI, Seshan VE, et al. Extrapleural pneumonectomy versus pleurectomy/decortication in the surgical management of malignant pleural mesothelioma: results in 663 patients. J Thorac Cardiovasc Surg 2008; 135: 620-26.

9 Lang-Lazdunski L, Bille A, Lal R, et al. Pleurectomy/decortication is superior to extrapleural pneumonectomy in the multimodality management of patients with malignant pleural mesothelioma. $J$ Thorac Oncol 2012; 7: 737-43.

10 Teh E, Fiorentino F, Tan C, Treasure T. A systematic review of lung-sparing extirpative surgery for pleural mesothelioma. $J$ R Soc Med 2011; 104: 69-80.

11 Nakas A, Martin Ucar AE, Edwards JG, Waller DA. The role of video 35 assisted thoracoscopic pleurectomy/decortication in the therapeutic management of malignant pleural mesothelioma. Eur J Cardiothorac Surg 2008; 33: 83-88.

12 Halstead JC, Lim E, Venkateswaran RM, Charman SC, Goddard M, Ritchie AJ. Improved survival with VATS pleurectomy-decortication in advanced malignant mesothelioma. Eur J Surg Oncol 2005; 31: 314-20.

13 Curran D, Sahmoud T, Therasse P, van Meerbeeck J, Postmus PE, Giaccone G. Prognostic factors in patients with pleural mesothelioma: the European Organization for Research and Treatment of Cancer experience. J Clin Oncol 1998; 16: 145-52.
114 Rusch VW. A proposed new international TNM staging system for malignant pleural mesothelioma from the International Mesothelioma Interest Group. Chest 1995; 108: 1122-28.

15 Kind P. The EuroQoL instrument: an index of health-related quality of life. In: Spilker B, ed. Quality of life and pharmacoeconomis in clinical trials, 2nd edn. Philadelphia, PA: Lippincott-Raven Publishers, 1996: 191-201.

16 Aaronson NK, Ahmedzai S, Bergman B, et al. The European Organization for Research and Treatment of Cancer QLQ-C30: a quality-of-life instrument for use in international clinical trials in oncology. J Natl Cancer Inst 1993; 85: 365-76.

17 Cantó A, Guijarro R, Arnau A, Galbis J, Martorell M,

10 García Aguado R. Videothoracoscopy in the diagnosis and treatment of malignant pleural mesothelioma with associated pleural effusions. Thorac Cardiovasc Surg 1997; 45: 16-19.

18 Curtis L, ed. Unit costs of health and social care 2011. Canterbury, Kent: Personal and Social Services Research Unit, University of Kent, 2011

19 Dolan P. Modeling valuations for EuroQol health states. Med Care 1997: 35: 1095-108

20 Lydick E, Epstein RS, Himmelberger D, White CJ. Area under the curve: a metric for patient subjective responses in episodic diseases. Qual Life Res 1995; 4: 41-45.

21 Cao C, Tian DH, Pataky KA, Yan TD. Systematic review of pleurectomy in the treatment of malignant pleural mesothelioma. Lung Cancer 2013; 81: 319-27.

22 Pickard AS, Neary MP, Cella D. Estimation of minimally important differences in EQ-5D utility and VAS scores in cancer. Health Qual Life Outcomes 2007; 5: 70.

23 Antunes G, Neville E, Duffy J, Ali N, and the Pleural Diseases Group, Standards of Care Committee, British Thoracic Society. BTS guidelines for the management of malignant pleural effusions. Thorax 2003; 58 (suppl 2): ii29-38.

24 Rahman NM, Ali NJ, Brown G, et al, and the British Thoracic Society Pleural Disease Guideline Group. Local anaesthetic thoracoscopy: British Thoracic Society Pleural Disease Guideline 2010. Thorax 2010; 65 (suppl 2): ii54-60

25 Rodriguez-Panadero F, Montes-Worboys A. Mechanisms of pleurodesis. Respiration 2012; 83: 91-98.

26 Bielsa S, Hernández P, Rodriguez-Panadero F, Taberner T, Salud A Porcel JM. Tumor type influences the effectiveness of pleurodesis in malignant effusions. Lung 2011; 189: 151-55.

27 Fysh ET, Tan SK, Read CA, et al. Pleurodesis outcome in malignant pleural mesothelioma. Thorax 2013; 68: 594-96.

28 American Thoracic Society. Management of malignant pleural effusions. Am J Respir Crit Care Med 2000; 162: 1987-2001.

29 National Institute for Health and Care Excellence. NICE consults on new draft recommendations for lung cancer drug. http://www.nice. org.uk/newsroom/pressreleases/CrizotinibACD.jsp (accessed Sept 15, 2013).

30 Asbestos Diseases Research Institute. Guidelines for the diagnosis and treatment of malignant pleural mesothelioma. Sydney, Australia: Asbestos Diseases Research Institute, 2013.

31 British Thoracic Society Standards of Care Committee. BTS statement on malignant mesothelioma in the UK, 2007. Thorax 2007; 62 (suppl 2): ii1-19. 